\title{
Adjuvant Anthracyclines and Taxanes: Quantifying the Costs of Therapeutic Advances in Breast Cancer
}

\author{
Patrick G. Morris $^{\mathrm{a}}$ Peter B. Bach ${ }^{\mathrm{b}}$ \\ ${ }^{\text {a }}$ Department of Medicine, \\ ${ }^{\mathrm{b}}$ Department of Epidemiology and Biostatistics, Memorial Sloan-Kettering Cancer Center, New York, NY, USA
}

Breast cancer represents a significant public health burden with over one million new cases diagnosed worldwide annually [1]. Since the development of adjuvant chemotherapy with cyclophosphamide, methotrexate and fluorouracil (CMF) over 30 years ago, successive advances in systemic therapy have incrementally improved the outcome of patients with early-stage disease. However the newer adjuvant chemotherapy regimens are also, in general, more expensive. This trend for increasing costs appears to be outstripping the corresponding benefits of each new therapy, making consecutive therapeutic advances less cost-effective [2]. In the current era of rising health-care costs coupled with global financial constraints there is increased scrutiny of the balance between the expenditure and improved efficacy of each therapy. In general, the assessment of the incremental cost-effectiveness of newer chemotherapy agents, targeted therapies and supportive medications has lagged behind their introduction.

Since the 1970s multiple randomized control trials have proven the efficacy of a variety of cytotoxic agents, including anthracyclines and taxanes. The Early Breast Cancer Trialists' Collaborative Group analyzed data from 14,000 women and demonstrated that the survival advantage of anthracyclinebased chemotherapy over CMF-like regimens was $4.2 \%$ at 10 years $(p<0.00001)$ [3]. Building on this foundation, several large clinical trials have shown the additional benefit of adjuvant taxanes. Overall the incorporation of taxanes into anthracycline-based regimens has improved 5-year survival by a further $3 \%$, based on a meta-analysis of over 22,000 patients [4]. Therefore sequential anthracycline-taxane combinations have become the standard of care for high-risk patients with early breast cancer. Although efficacy is well established, far less is known about the cost-effectiveness of this approach. A recent meta-analysis suggested there was considerable variation between the cost-effectiveness of taxane-based chemotherapies, depending on the use of either paclitaxel or docetaxel, different dosing schedules and possible inconsistencies in efficacy among certain patient subgroups [5]. Therefore, further research is needed to quantify the costs of new treatments and to put this in context with the associated benefits.

In this issue of the journal Braun et al. describe a cost analysis from a randomized phase III study, which compared sequential anthracycline-taxane chemotherapy with CMF for women with node-positive early breast cancer [6]. Patients received either 4 cycles of epirubicin and cyclophosphamide (EC) followed by 4 cycles of docetaxel (DOC) both given every 3 weeks or 6 cycles of intravenous CMF given on days 1 and 8 every 4 weeks. A detailed analysis of 110 patients including quantification of the direct costs of chemotherapy, as well as indirect costs showed that the mean cost for sequential EC-DOC was $€ 8,459$, meaningfully higher than $€ 4,973$ for CMF. Cytotoxic drugs contributed $67 \%$ of the cost of the anthracycline-taxane combination and the mean cost per patient of chemotherapy was $€ 5,673$ for EC-DOC compared to only $€ 75$ for CMF, which was somewhat offset by the staff costs associated with the greater number of days of chemotherapy administration in the group receiving CMF (12 versus 8). As expected EC-DOC was associated with a greater number of hospitalizations, but the mean cost of each hospitalization was higher in the CMF group, possibly as a result of greater G-CSF use. The major strength of this report is the highly detailed cost quantification, incorporating different aspects of the administration of chemotherapy. This may allow broader interpretation of the results outside the particular heath-care system studied.

However this study does not provide all the answers regarding the impact of the incremental cost of incorporating docetaxel into adjuvant chemotherapy. The two groups were randomized to different numbers of chemotherapy cycles ( 8 versus 6 ), so the interpretation of costs is confounded by the number of treatment cycles. In addition the specific study

\section{KARGER}

Fax +497614520714

Information@Karger.de

www.karger.com (c) 2009 S. Karger GmbH, Freiburg

Accessible online at:

www.karger.com/onk
Dr. Peter B. Bach MD, MAPP

Department of Epidemiology and Biostatistics

Memorial Sloan-Kettering Cancer Cente

307 E $63^{\text {rd }}$ Street, mailbox 221, New York, NY 10065, USA

Tel. +1 646 735-8137, Fax -0011

bachp@mskcc.org 
regimens differed by both taxane and anthracycline since anthracycline-taxane chemotherapy (EC-DOC) was compared to an older non-anthracycline, non-taxane regimen (CMF). To determine the true contribution of taxanes to the cost of adjuvant chemotherapy it would be necessary to compare costs of an anthracycline-taxane combination with an anthracycline regimen without taxanes. Such an analysis is beyond the scope of the current report but could be performed from a later cohort of patients from the same randomized trial since the anthracycline-based FEC regimen was compared to EC-DOC.

In the future the detailed methodology described by Braun et al. could be used to quantify the costs of other taxanebased regimens. In terms of taxane-scheduling an analysis of weekly paclitaxel compared to docetaxel every 3 weeks would be interesting since drug acquisition costs are substantially lower for paclitaxel than for docetaxel, and these two taxane schedules have recently demonstrated equivalent efficacy [7, 8]. Likewise there are scant data on the costs of dose-dense chemotherapy, in which treatment is administered at shorter intervals and which has been shown to improve survival [9]. Although dose-dense anthracycline-taxane therapy requires growth factor-support, which increases costs, this is potentially offset by the lower rate of hospitalization for febrile neutropenia among patients receiving dose-dense therapy $[9,10]$.

The costs of adjuvant breast cancer treatments have also risen since the introduction of trastuzumab, which has increased survival for patients with early breast cancer that overexpresses the human epidermal growth factor receptor 2 (HER2) [11-14]. A relatively higher proportion of younger patients have tumors that overexpress HER2 and so the increased cost of adjuvant trastuzumab needs to be balanced against the potentially greater gain in quality-adjusted life years (QALY) for this group compared to an older patient cohort $[15,16]$. In addition the use of adjuvant trastuzumab could potentially reduce the cost of expensive treatments in the metastatic setting due to the resultant lower rate of relapse [15].

The targeting of drugs to HER2 is an example of how adjuvant therapy is increasingly tailored to individual tumor biology. Subgroup analyses from clinical trials have suggested that some patients with endocrine-responsive disease may derive less benefit from taxanes [17]. Therefore more granular risk stratification techniques are needed, particularly in settings where the potential benefits of adjuvant therapy are more uncertain. One innovation, which may illustrate a way forward, is the Oncotype DX ${ }^{\mathrm{TM}}$ (Genomic health Inc., Redwood City, CA, USA) gene assay, which evaluates tumor expression of 16 cancer-related genes relative to 5 reference genes and offers an individualized assessment of prognosis in addition to predicting response to chemotherapy [18]. This risk stratification technique may help refine adjuvant chemotherapy recommendations and allow a reduction in costs by better targeting of therapy to those most likely to benefit. Additionally as patients are increasingly involved in treatment decisions there is an unmet need to combine quality-of-life assessments with economic evaluations of therapeutic innovations. In the future it is hoped that cost analyses as described by Braun et al. will play an integral part in the assessment of therapeutic advances and that detailed cost-effectiveness analyses can parallel improvements in individualization of adjuvant therapy based on tumor biology.

\section{Conflict of Interest}

Dr. Morris reports receiving honoraria from Eisai, Genomic Health, Pfizer, Haymarket Media, Bristol-Myers Squibb, Genentech, Novartis and OrthoBiotech.

Dr. Bach reports receiving consulting fees from Genentech, Wyeth, and Abraxis and lecture fees from Genentech, Biogen Idec, and Johnson \& Johnson.

\section{References}

1 Stewart BW KP: World cancer report; Iarc press, 2003

2 Bach PB: Limits on medicare's ability to control rising spending on cancer drugs. N Engl J Med 2009; 360:626-633.

$>3$ Early Breast Cancer Trialists' Collaborative Group (EBCTCG): Effects of chemotherapy and hormonal therapy for early breast cancer on recurrence and 15-year survival: An overview of the randomised trials. Lancet 2005;365:1687-1717.

4 De Laurentiis M, Cancello G,D'Agostino D, Giuliano M, Giordano A, Montagna E, Lauria R, Forestieri V, Esposito A, Silvestro L, Pennacchio R, Criscitiello C, Montanino A, Limite G, Bianco AR, De Placido S: Taxane-based combinations as adjuvant chemotherapy of early breast cancer: A metaanalysis of randomized trials. J Clin Oncol 2008;26: $44-53$.
5 Ward S, Simpson E, Davis S, Hind D, Rees A, Wilkinson A:Taxanes for the adjuvant treatment of early breast cancer: Systematic review and economic evaluation. Health Technol Assess 2007;11:1-144.

-6 Braun M, Jacobs VR, Wagenpfeil S, Sattler D, Harbeck N, Nitz U, Bernard R, Kuhn W, IhbeHeffinger A: Cost analysis comparing an anthracycline/docetaxel regimen to CMF in patients with early stage breast cancer. Onkologie 2009;32: 473-481.

7 Sparano JA, Wang M, Martino S, Jones V, Perez EA, Saphner T, Wolff AC, Sledge GW, Jr., Wood WC, Davidson NE: Weekly paclitaxel in the adjuvant treatment of breast cancer. N Engl J Med 2008; 358:1663-1671.

8 Vu T, Ellard S, Speers CH, Taylor SC, de Lemos ML, Hu F, Kuik K, Olivotto IA: Survival outcome and cost-effectiveness with docetaxel and paclitaxel in patients with metastatic breast cancer: A population-based evaluation. Ann Oncol 2008;19:461-464.
9 Citron ML, Berry DA, Cirrincione C, Hudis C Winer EP, Gradishar WJ, Davidson NE, Martino S, Livingston R, Ingle JN, Perez EA, Carpenter J, Hurd D, Holland JF, Smith BL, Sartor CI, Leung EH, Abrams J, Schilsky RL, Muss HB, Norton L: Randomized trial of dose-dense versus conventionally scheduled and sequential versus concurrent combination chemotherapy as postoperative adjuvant treatment of node-positive primary breast cancer: First report of intergroup trial c9741/cancer and leukemia group B trial 9741. J Clin Oncol 2003; 21:1431-1439.

10 Burnell MJ, Levine MN, Chapman JA, Bramwell V, Vandenberg T, Chalchal HI, Albain K, Perez E, Rugo H, Pritchard K: A phase III adjuvant trial of sequenced ec + filgrastim + epoetin-alpha followed by paclitaxel compared to sequenced ac followed by paclitaxel compared to $\mathrm{CEF}$ in women with nodepositive or high-risk node-negative breast cancer (ncic ctg ma.21). Proc Am Soc Clin Oncol 2007; 25:Abstr. 550 
11 Romond EH, Perez EA, Bryant J, Suman VJ, Geyer CE, Jr., Davidson NE, Tan-Chiu E, Martino S, Paik S, Kaufman PA, Swain SM, Pisansky TM, Fehrenbacher L, Kutteh LA, Vogel VG, Visscher DW, Yothers G, Jenkins RB, Brown AM, Dakhil SR, Mamounas EP, Lingle WL, Klein PM, Ingle JN, Wolmark N: Trastuzumab plus adjuvant chemotherapy for operable HER2-positive breast cancer. N Engl J Med 2005;353:1673-1684.

12 Slamon D, Eiermann W, Robert N, Pienkowski T, Martin M, Pawlicki M, Chan A, Smylie M, Liu M, Falkson C, Pinter T, Fornander T, Shiftan T, Valero V, Mackey J, Tabah-Fisch I, Buyse M, Lindsay M, Riva A, Bee V, Pegram M, Press M, Crown J: BCIRG 006: 2nd interim analysis phase III randomized trial comparing doxorubicin and cyclophosphamide followed by docetaxel (ACT) with doxorubicin and cyclophosphamide followed by docetaxel and trastuzumab (ACTH) with docetaxel, carboplatin and trastuzumab $(\mathrm{TCH})$ in HER2neu positive early breast cancer patients. San Antonio Breast Cancer Symposium 2006:Late Breaking Abstr. 52.
13 Joensuu H, Kellokumpu-Lehtinen PL, Bono P, Alanko T, Kataja V, Asola R, Utriainen T, Kokko R, Hemminki A, Tarkkanen M, Turpeenniemi-Hujanen T, Jyrkkio S, Flander M, Helle L, Ingalsuo S, Johansson K, Jaaskelainen AS, Pajunen M, Rauhala M, Kaleva-Kerola J, Salminen T, Leinonen M, Elomaa I, Isola J: Adjuvant docetaxel or vinorelbine with or without trastuzumab for breast cancer. $\mathrm{N}$ Engl J Med 2006;354:809-820.

14 Piccart-Gebhart MJ, Procter M, Leyland-Jones B, Goldhirsch A, Untch M, Smith I, Gianni L, Baselga J, Bell R, Jackisch C, Cameron D, Dowsett M, Barrios CH, Steger G, Huang CS, Andersson M, Inbar M, Lichinitser M, Lang I, Nitz U, Iwata H, Thomssen C, Lohrisch C, Suter TM, Ruschoff J, Suto T, Greatorex V, Ward C, Straehle C, McFadden E, Dolci MS, Gelber RD: Trastuzumab after adjuvant chemotherapy in HER2-positive breast cancer. N Engl J Med 2005;353:1659-1672.
15 O'Cearbhaill R, Wilson E, DeFrein A, Qadir Z, McDonnell D, Crown J: Estimating the real cost of adjuvant trastuzumab in patients with HER-2+ early stage breast cancer: potential impact of reduced use of expensive oncology drugs in metastatic breast cancer. Proc Am Soc Clin Oncol 2007; Abstr. 6534.

16 Hudis CA: Trastuzumab-mechanism of action and use in clinical practice. N Engl J Med 2007;357:39-51.

17 Hayes DF, Thor AD, Dressler LG, Weaver D, Edgerton S, Cowan D, Broadwater G, Goldstein LJ, Martino S, Ingle JN, Henderson IC, Norton L, Winer EP, Hudis CA, Ellis MJ, Berry DA: HER2 and response to paclitaxel in node-positive breast cancer. N Engl J Med 2007;357:1496-1506.

18 Paik S, Tang G, Shak S, Kim C, Baker J, Kim W, Cronin M, Baehner FL, Watson D, Bryant J, Costantino JP, Geyer CE, Jr., Wickerham DL, Wolmark $\mathrm{N}$ : Gene expression and benefit of chemotherapy in women with node-negative, estrogen receptor-positive breast cancer. J Clin Oncol 2006;24:3726-3734. 